\title{
OTIOTOMTCS
}

Revista de economía, empresa y sociedad

BLOCKCHAIN, INTELIGENCIA ARTIFICIAL, 3D PRINTING

\section{Innovaciones tecnológicas en la cadena de suministro aplicadas al eGommerce}

\section{Xavier Budet Jofra}

Profesor colaborador del máster de Dirección de operaciones y logística integral (UOC)

\section{Alexis Pérez Gómez}

Profesor colaborador del máster de Dirección de operaciones y logística integral (UOC)

RESUMEN En los últimos años se han vertebrado soluciones tecnológicas de menor a mayor complejidad que fomentan la evolución de la logística y de la cadena de suministro y que permiten la comunión entre requisitos y limitaciones que los clientas sufren en su proceso de compra en la eCommerce.

El adelanto de ciertas tecnologías en otros sectores ha permitido su incorporación a la logística y a la cadena de suministro añadiendo valor, no solo al cliente mediante la personalización de la oferta de productos y servicios, sino también influyendo a proveedores y otros actores que componen la cadena, dotando de agilidad en la planificación, gestión y transporte de inventario.

En el futuro, la adopción de estas nuevas tecnologías requerirá de un proceso de especialización en detrimento del rol puramente operacional que ha caracterizado hasta ahora al sector logístico, así como de la generación de nuevos estándares y de entidades reguladoras que permitan su integración y su desarrollo.

PALABRAS CLAVE eCommerce; logística; cadena de suministro; internet of things; big data; blockchain; drones; inteligencia artificial; 3D printing 


\title{
Technological supply chain innovations applied to eCommerce
}

\begin{abstract}
In recent years, technological solutions of varying degrees of complexity have emerged that have driven the evolution of logistics and supply chain, and which enable concordance between the requirements and limitations faced by customers in the eCommerce shopping process.

Advances in certain technologies in other sectors has enabled them to be incorporated into logistics and the supply chain, not only adding value for the customer through the customization of the products and services on offer, but also having an impact on suppliers and other actors that form part of the chain, enabling them to achieve enhanced responsiveness in terms of stock planning, management and transportation.

In the future, the adoption of these new technologies will require a process of specialization at the expense of the purely operational role that has characterized the logistics sector to date, as well as requiring new standards and regulatory bodies to be generated that enable their integration and development.
\end{abstract}

KEYWORDS eCommerce; logistics; supply chain; Internet of Things; Big Data; blockchain; drones; artificial intelligence; 3D printing

\section{Introducción}

En el artículo «La logística como fuente de valor añadido al eCommerce» (Budet y Pérez, 2018) queda patente, por un lado, la variabilidad y diversidad de expectativas de clientes fruto de la adopción del eCommerce como solución a sus necesidades y, por otro lado, las limitaciones inherentes a las plataformas logísticas existentes. La no comunión entre requisitos y limitaciones mencionados puede derivar a los problemas propios de la última milla. Para afrontar tal cosa, en los últimos años se han vertebrado soluciones tecnológicas de menor a mayor complejidad que, a la vez, fomentan la evolución de la logística y de la cadena de suministro.

Se empieza a hablar de la cadena de suministro 4.0, es decir, una cadena de suministro completamente digitalizada con flujos de información integrados en toda la cadena donde esta información se supone fiable y a tiempo real, y que se ha convertido en un factor básico para poder tomar decisiones de una forma predecible, autónoma y consistente. La tecnología tiene que permitir, por un lado, capturar la información a tiempo real, compartirla, tratarla, analizarla, establecer 
tendencias y patrones y, por otro lado, automatizarla para optimizar la planificación, facilitar la gestión y acelerar la ejecución de las tácticas y acciones operativas derivando en procesos más flexibles, rápidos, eficaces y eficientes.

En más detalle, se ha vivido la transición desde la implementación de soluciones clásicas, como la ya normalizada trazabilidad de envíos, hacia la opción de entrega mediante múltiples canales de abastecimiento (omnicanalidad), entregas en el mismo día con hora acordada, acercar el inventario físicamente antes de ejecutar la orden de compra (modelos predictivos), garantizar la entrega 24/365 vía puntos de recogida, la manufactura en 3D del bien comprado o la entrega deslocalizada mediante drones o vehículos autónomos. Todos ellos son claros ejemplos de cómo la innovación permite cerrar el círculo virtuoso al eCommerce para que la logística añada valor en la experiencia de usuario y reduzca ineficiencias operacionales derivadas de múltiples intentos de entrega o regresos debido a entregas fallidas.

En la figura 1 se muestran soluciones tecnológicas que están ganando un espacio en la innovación de la cadena de suministro y también otras consolidadas.

Figura 1. Ciclo de sobreexpectación de las tecnologías emergentes
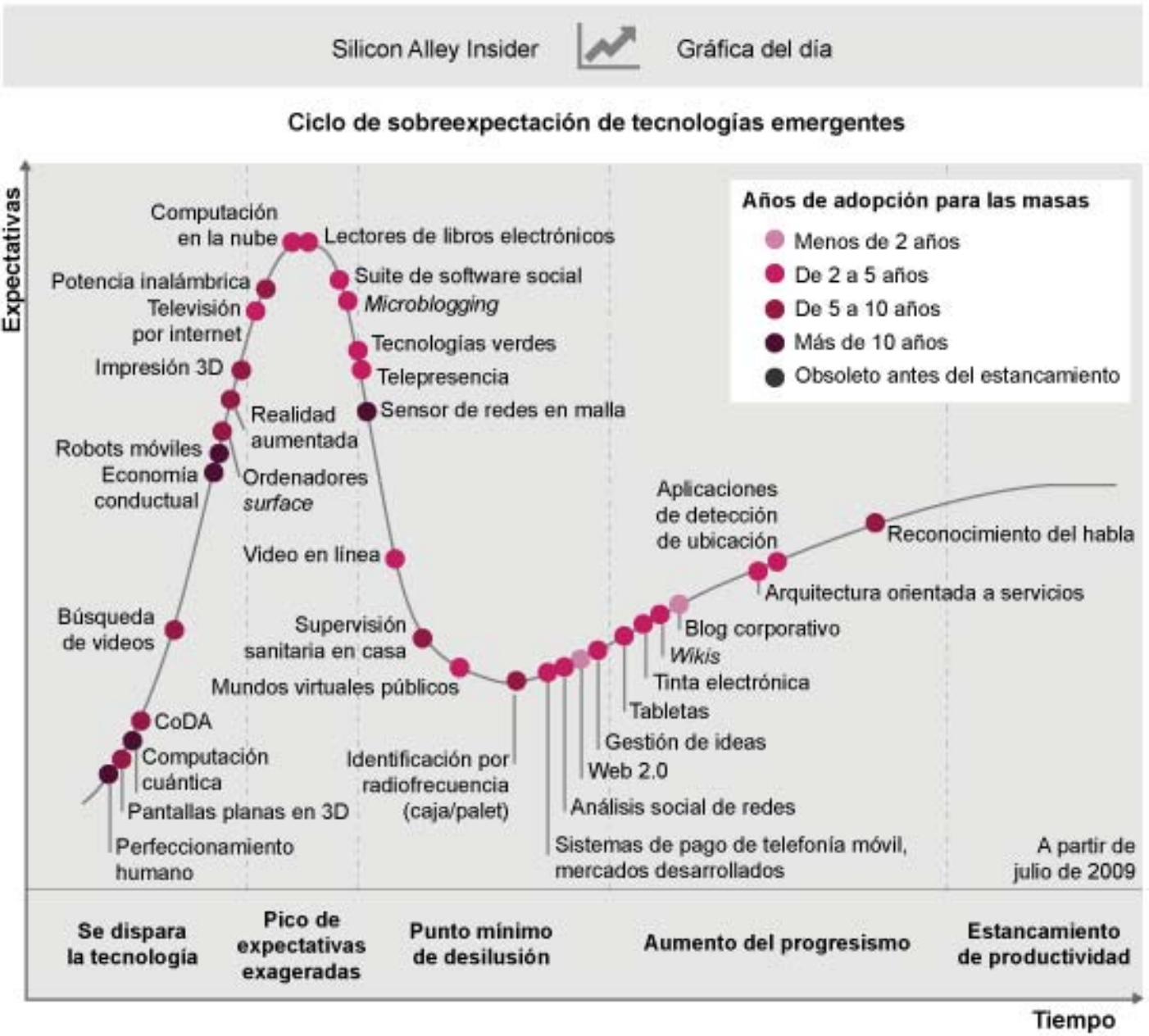

Fuente: Jeff McNeil, 2011 https://commons.wikimedia.org/wiki/File\%3AGartner_Hype_Cycle_for_Emerging_Technologies.gif. 
En el presente artículo nos centraremos en la descripción de un conjunto de tecnologías que están adquiriendo cada vez más relevancia en la cadena de suministro y en su recorrido en los próximos años, de acuerdo a los requerimientos del canal del eCommerce. Tecnologías que presentan ventajas como, por ejemplo: la mejora operacional mediante la democratización de servicios de la mano del paradigma blockchain con el objetivo de fomentar la competitividad en la calidad de servicios entre proveedores logísticos que se ofrecen para un mismo servicio logístico; o bien, la anticipación de la demanda mediante modelos predictivos; el incremento del éxito en las entregas mediante sistemas de entregas automatizadas; o el acercamiento de la producción al cliente final mediante la impresión en 3D.

\section{Blockchain como democratización en la subcontratación de servicios logísticos}

Esta tecnología se basa en la distribución de la información en lugar de copiarla o duplicar el contenido para su procesado. Sus orígenes de uso están estrechamente relacionados con las criptomonedas, pero se podrían diversificar las aplicaciones dadas las ventajas que día a día se descubren en relación con su uso.

Figura 2. Proceso blockchain

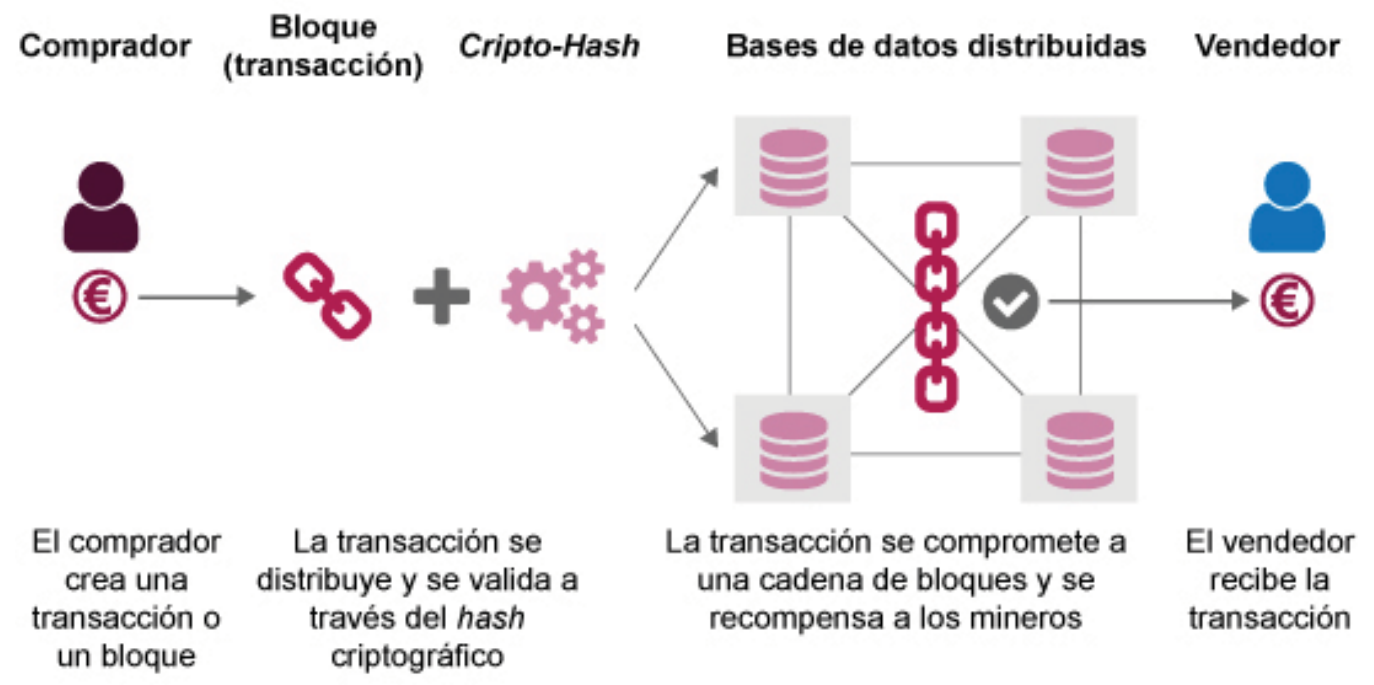

Fuente: B140970324 https://commons.wikimedia.org/wiki/File\%3ABlockchain-Process.png

La premisa principal se basa en tratar la información de una forma descentralizada, compartida y continuamente reconciliada a través de bases de datos. Esta información se distribuye a muchos ordenadores simultáneamente, pero a la vez es accesible para cualquier persona con acceso a internet. De este modo, se evita que el contenido íntegro esté expuesto a vulnerabilidades, como por ejemplo ataques 
cibernéticos, o incluso excluyendo las entidades reguladoras de su gestión para que no ejerzan un abuso de poder.

Un ejemplo sería el caso de las criptomonedas. Bitcoin es una criptomoneda y a la vez una red que gestiona, de manera descentralizada, todas las transacciones que suceden con esta moneda. De este modo se evita la dependencia de una autoridad central o entidad reguladora que centralice su gestión.

Al llevar esta filosofía a la logística, una de las utilidades más claras es la de garantizar la trazabilidad de cualquier producto en la cadena de suministro y, junto con la información proveniente de sensores (IoT), garantizar que un producto no ha perdido en ningún momento la cadena de frío.

Otra utilidad consistiría, en primer lugar, en centralizar toda la variabilidad de combinaciones y escenarios de los múltiples elementos de la cadena de suministro a los cuales un envío puede estar expuesto. Seguidamente, haciendo uso de la tecnología RFID en las cajas, estas podrían comunicar la necesidad de desplazamiento de $A$ a $B$ y una fecha de entrega concreta en la red. En esta fase, se descentralizaría entre los múltiples operadores logísticos los detalles de la entrega para poder competir por la potestad del envío en función de sus capacidades reales de entrega, disponibilidad y condiciones. Con este cómputo de transacciones, la tarjeta RFID otorgaría el envío al proveedor logístico que mejor se adaptara a los requisitos del cliente en cuanto a precio y calidad de servicio.

A pesar de los beneficios que se entrevén de esta tecnología, hay varios puntos para aclarar. Blockchain se basa en una serie de nodos que permiten la replicación y autenticación de la información; hace falta todavía aclarar cuáles tienen que ser estos nodos, cómo se compartirá la información en la cadena de suministro y el hardware (discos, ancho de banda) necesario para guardar la ingente cantidad de información a almacenar. Por otro lado, la seguridad de las redes many-to-many todavía está en proceso experimental. Finalmente, hay que tener en cuenta la carencia de escalabilidad de este tipo de soluciones dada la falta de adopción de la tecnología blockchain entre los diferentes integrantes de la cadena de suministro. Además de la colaboración necesaria, hará falta que lo adopten muchas más compañías para crear estándares que la hagan más asequible y eficaz.

\section{Inteligencia artificial: sistemas recomendadores y modelos predictivos}

El término internet of things (abreviado generalmente loT) se refiere al envío de datos entre dispositivos y/o máquinas para mejorar la ejecución de la cadena de suministro.

La continua y creciente penetración de internet en los hogares (llegando al 66,4 \% de media mundial en el año 2017) habilita la evolución del eCommerce gracias al paradigma del «internet de las cosas» hacia plataformas inteligentes que 
ofrecen servicios más aptos a los perfiles de sus clientes. En consecuencia, permite conocer mejor la potencial demanda e incluso predecir los niveles de inventario que harán falta para garantizar la calidad de servicio de la misma.

Figura 3. Penetración de internet

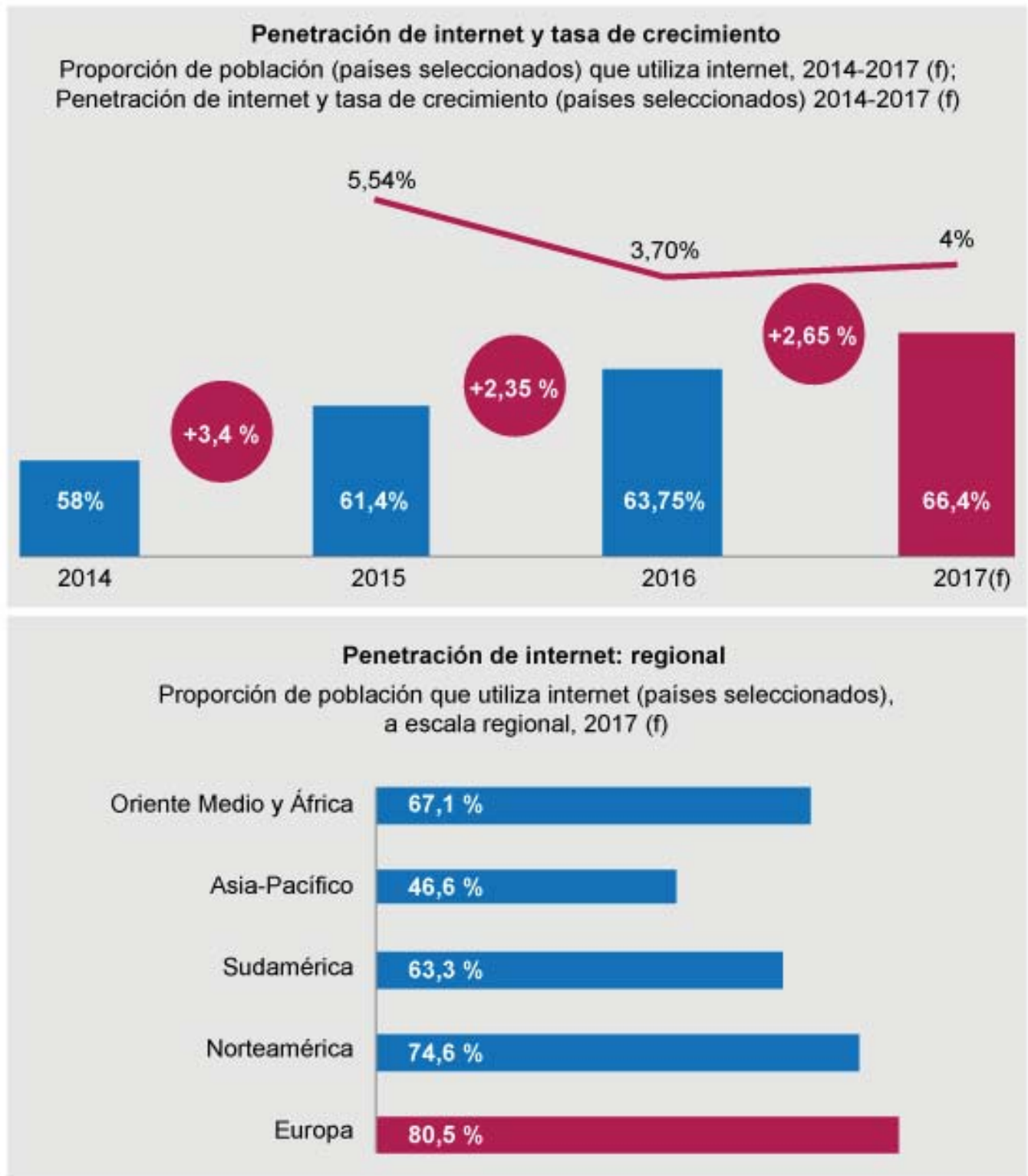

Fuente: www.ecommercefoundation.org/reports

El loT permitirá unas operaciones más eficientes combinando sensores, cámaras, máquinas, software, bases de datos e internet para conectar el mundo físico y el mundo digital, extrayendo información en tiempo real. Este entramado de dispositivos permitirá: mejorar los inputs para la cadena de suministro que facilitará la automatización y gestión de datos para realizar posteriores análisis predictivos o prescriptivos, reduciendo los errores humanos; crear alarmas para mejorar la seguridad; monitorizar procesos complejos; optimizar rutas y entregas de una forma 
automática; posicionar y planificar inventarios (sobre la base de la demanda); controlar la cadena de frío y la trazabilidad de los productos, etc.

La consultora Gartner estima que en 2020 habrá 26.000 millones de dispositivos conectados a internet que generarán, aproximadamente, 300.000 millones de datos. Hay que reflexionar con cuidado sobre esta gran cantidad de datos que, muy probablemente, crecerá exponencialmente: sobre dónde y cómo guardarlos, cuánto tiempo, cómo y cuáles tendrán que ser gestionados, cuáles habrá que tener de forma instantánea, cómo se garantizará la seguridad de estos datos, cómo se hará su integración, qué infraestructuras de red se necesitarán, etc. Recoger, guardar y analizar toda esta información requerirá diferentes procesos, habilidades y tecnologías. En un futuro inmediato, adquirir estas habilidades y tecnologías será clave para que una empresa esté en condiciones de competir en los dos ejes diferenciadores de la cadena de suministro: calidad en el servicio y eficiencia.

De la gestión de la gran cantidad de datos que se generan actualmente se deriva el término big data, utilizado para describir la información almacenada en las bases de datos, tanto de una forma estructurada (como por ejemplo la información de una sistema de gestión de almacenes) como desestructurada (como por ejemplo los datos de las redes sociales o de un call center).

Se calcula que el $90 \%$ de los datos mundiales han sido creados solo en los dos últimos años y su crecimiento, como ya se ha dicho antes, es exponencial. Estos datos por sí solos no sirven para nada. Por lo tanto, hace falta discernir la información relevante de la que no lo es para poder convertirla en mejores decisiones que optimicen la cadena de suministro. También existe información aparentemente no útil que, con la tecnología adecuada, nos puede ayudar a establecer patrones y relaciones para poder modelar mejor la demanda y planificar eficazmente las operaciones. Es en este punto donde la inteligencia artificial está tomando cada vez más relevancia para poder automatizar el tratamiento de estos datos con sistemas capaces de «autoaprendizaje» que puedan afinar, con el tiempo, los análisis y optimizar las respuestas.

\subsection{Sistemas recomendadores}

Muchas plataformas de eCommerce utilizan recomendadores para ayudar a sus clientes a encontrar productos durante el proceso de compra. Un sistema recomendador aprende del cliente y sugiere qué productos encontrará que se adapten mejor a sus necesidades de entre todos los productos disponibles.

Por ejemplo, el sistema recomendador de Amazon se basa en las compras anteriores del cliente, otros productos existentes en el carro de la compra, artículos que el cliente ha marcado como favoritos con anterioridad, o artículos que clientes 
con similares patrones de conducta han marcado como interesantes durante su proceso de compra o han acabado comprando. La metodología consiste en construir grupos de productos en función de la relación existente entre los artículos. Cuando el cliente hace una compra, Amazon le recomendará otros artículos que pertenecen al mismo grupo del artículo comprado. Esta proximidad puede ser definida por categoría, uso, precio... y los candidatos propuestos se pueden observar en las zonas llamadas «artículos ver», «clientes que han visto este artículo vieron también...» o «frecuentemente comprado juntos».

La lógica de los recomendadores se basa en realizar nichos o «clústeres»; por un lado, de productos a la venta y, por otro lado, de grupos de usuario con interés parecido.

La pertenencia a cada uno de estos clústeres se basa en la correlación estadística de cada elemento que compone el grupo. Cada elemento se parametriza en campos que se van poblando con el histórico de compras, como por ejemplo precio, categorías de uso, zona geográfica con mayor venta, etc.

Cuando un cliente efectúa una compra de un nuevo producto, esta compra lo acerca a uno de estos nichos por vecindad a productos similares. En consecuencia, recibirá la recomendación de los productos del mismo clúster.

Figura 4. Concepto gráfico de sistema recomendador

Filtrado basado en el usuario

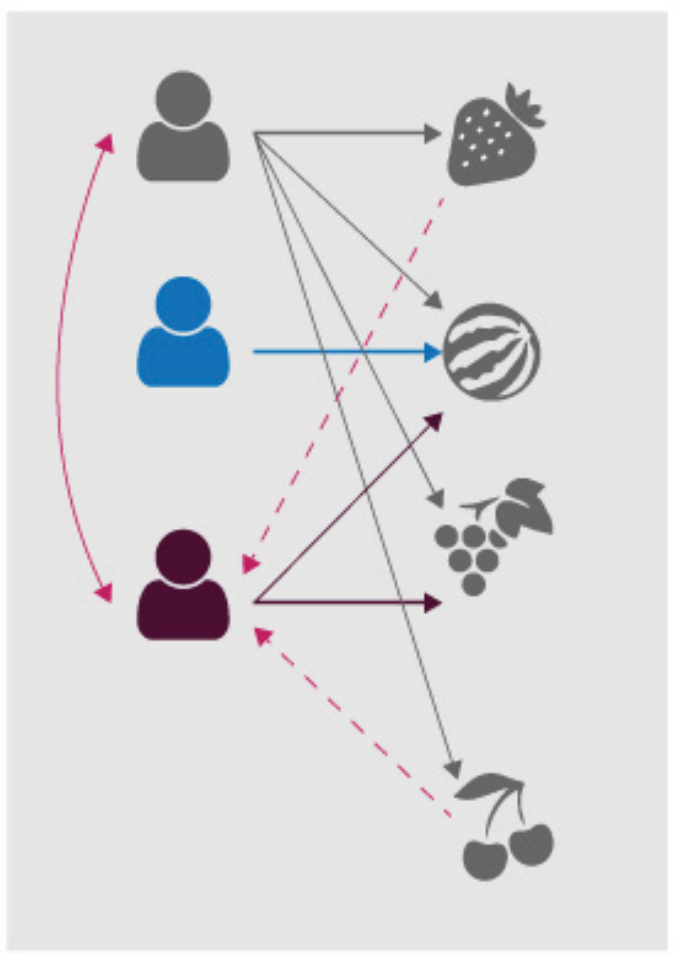

Filtrado basado en el elemento

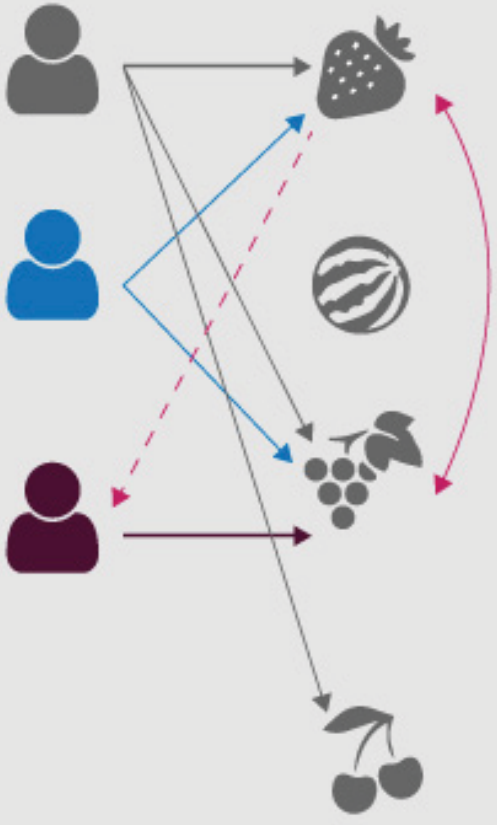


Asimismo, los recomendadores analizan el patrón de conducta del cliente y lo clasifican en nichos de clientes parecidos para recibir recomendaciones de compras antiguas de estos clientes «homólogos».

Se estima en un 12 \% la aportación de ingresos debido a los sistemas de recomendación, optimizando a la vez la conversión de visita a venta, con un factor multiplicador de 5,5 veces más grande hacia los clientes que no van a interactuar con recomendaciones. Comparados con sistemas de publicidad anteriores, los nuevos recomendadores son 1,7 veces más efectivos.

\subsection{Modelos predictivos}

Otra funcionalidad de la inteligencia artificial son los modelos predictivos que acercan el inventario a la demanda. Así, por ejemplo, Amazon, el gigante de Estados Unidos, entre otras patentes ha generado el método conocido como «envío anticipado" a la compra del cliente, mediante el cual balancea inventario entre centros de distribución cercanos al cliente, a partir de órdenes anteriores y otros factores que pueden indicar una alta probabilidad de la materialización de la compra. Esta parametrización se basa en el estudio del histórico de transacciones de otros clientes y de sus perfiles, del cual se identifica una cadena de sucesos. Es decir, es un modelo predictivo que aprende del histórico de información, y asigna altas probabilidades al hecho de que vuelva a suceder algo en función de su frecuencia de aparición en el pasado. Si un cliente está reproduciendo un patrón similar que ha sucedido en el pasado, habrá muchas probabilidades de que acabe desembocando en el mismo final: la compra. Esta predicción permite anticiparse a la compra empezando a mover inventario en función de los productos por los cuales se interese un cliente. También se puede llevar a otros ámbitos (comunidad, región o país) dependiendo de los patrones de conducta que se puedan identificar entre los datos existentes.

Los mecanismos para aprender de datos históricos «se entrenan» y automáticamente se comprueba su fiabilidad. Por ejemplo, se puede destinar un $70 \%$ de información histórica para hacer que el modelo predictivo «entrene y aprenda». Cuando hay ciertos patrones detectados, se comprueba la eficacia con el $30 \%$ de datos históricos restante; como se conoce el resultado final, pues se trata de sucesos del pasado, se puede contrastar si el resultado del modelo predictivo es correcto y el porcentaje de eficacia.

Gráficamente, el modelo predictivo de estructuras complejas de datos se puede representar con árboles de decisión donde cada rama, desde la raíz hasta un nodo final, respondería a un patrón con cierta probabilidad de éxito, medida por la cantidad de sucesos ocurridos en el pasado que siguen la rama en cuestión. 
Figura 5. Árbol de decisión usando un diagrama de flujo

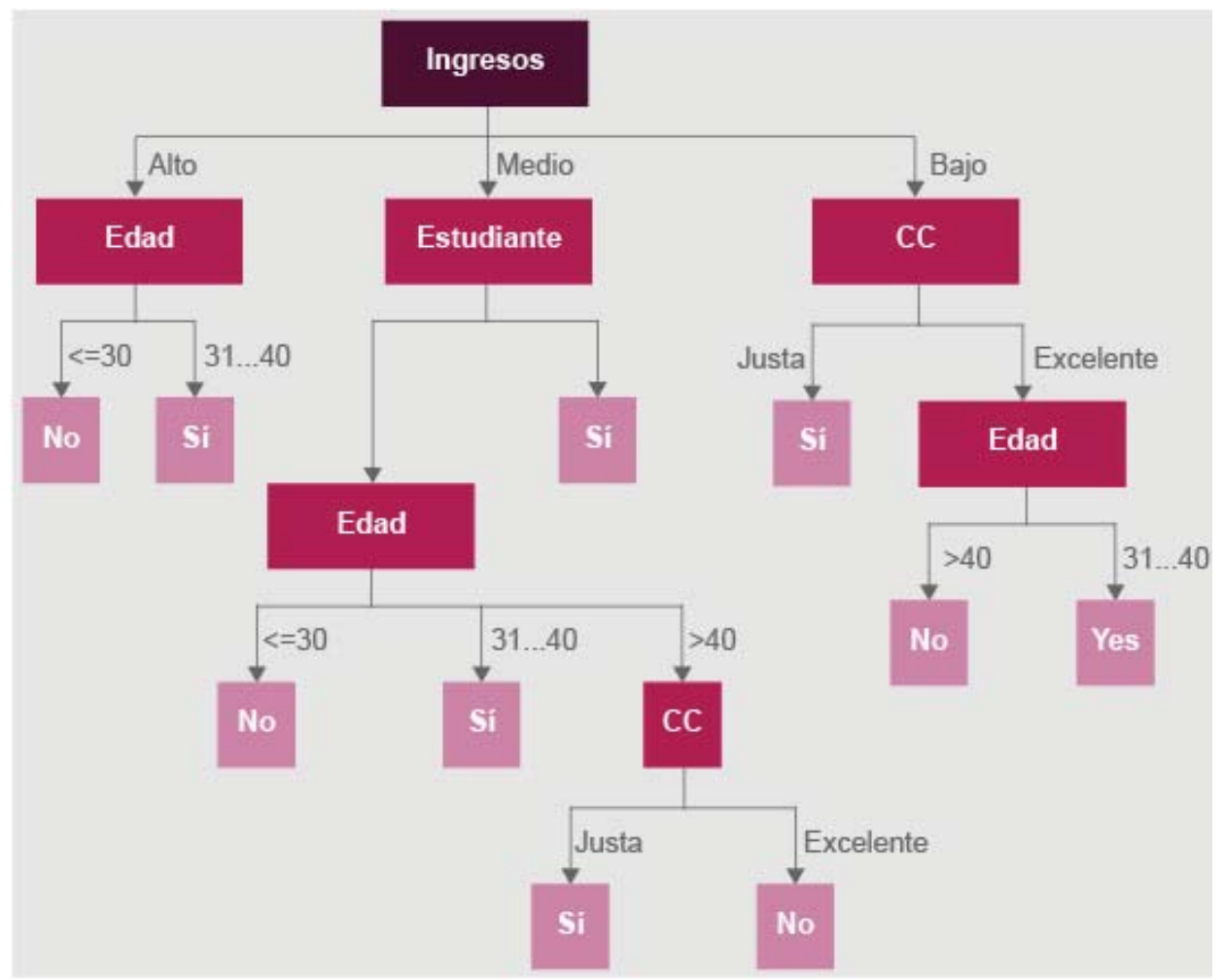

Fuente: adaptación de https://www.edureka.co/blog/decision-trees/

En la figura 5 se puede observar un modelo predictivo de compra de un cliente en función de parámetros más propios del individuo como la edad, si es estudiante o no, calidad financiera otorgada por la entidad de crédito y nivel salarial. Los nodos constituidos se convierten en frecuencia de aparición sobre la base de datos histórico, construido en el mencionado entrenamiento y validado con datos históricos no utilizados durante la generación de la lógica del modelo predictivo.

Este árbol de decisión sirve para establecer patrones de bajo qué premisas habrá probabilidad de demanda en una zona. Esto permite acercar inventario a los casos de clientes parecidos en cuanto a estos parámetros.

Estos modelos permiten tomar acciones de marketing y de publicidad hacia un cliente que cumple ciertos patrones que apuntan a una muy alta probabilidad de compra, ofreciéndole un último mensaje publicitario que pueda ayudar a materializar, en forma de compra, las interacciones del usuario con la plataforma web.

Amazon también se hace con las preferencias y hábitos de los clientes; en la anticipación de envío se intenta predecir qué querrá el cliente y enviarlo automáticamente previamente a su compra o petición. Gran parte de las compras son redundantes y, en caso de productos de primera necesidad, los patrones predictivos son robustos debido a las altas probabilidades asociadas a la repetitividad, lo que permite a Amazon asumir riesgos de inventario acercando los productos a los clientes cuando lo determinan estos patrones. Desgraciadamente, esta informa- 
ción también se puede usar para ajustar precios y jugar con la oferta y la demanda, o bien para decidir qué productos son aptos para promociones y cuáles otros pueden ser excluidos para poder sacar más margen.

Figura 6. Captura de patente Amazon anticipate

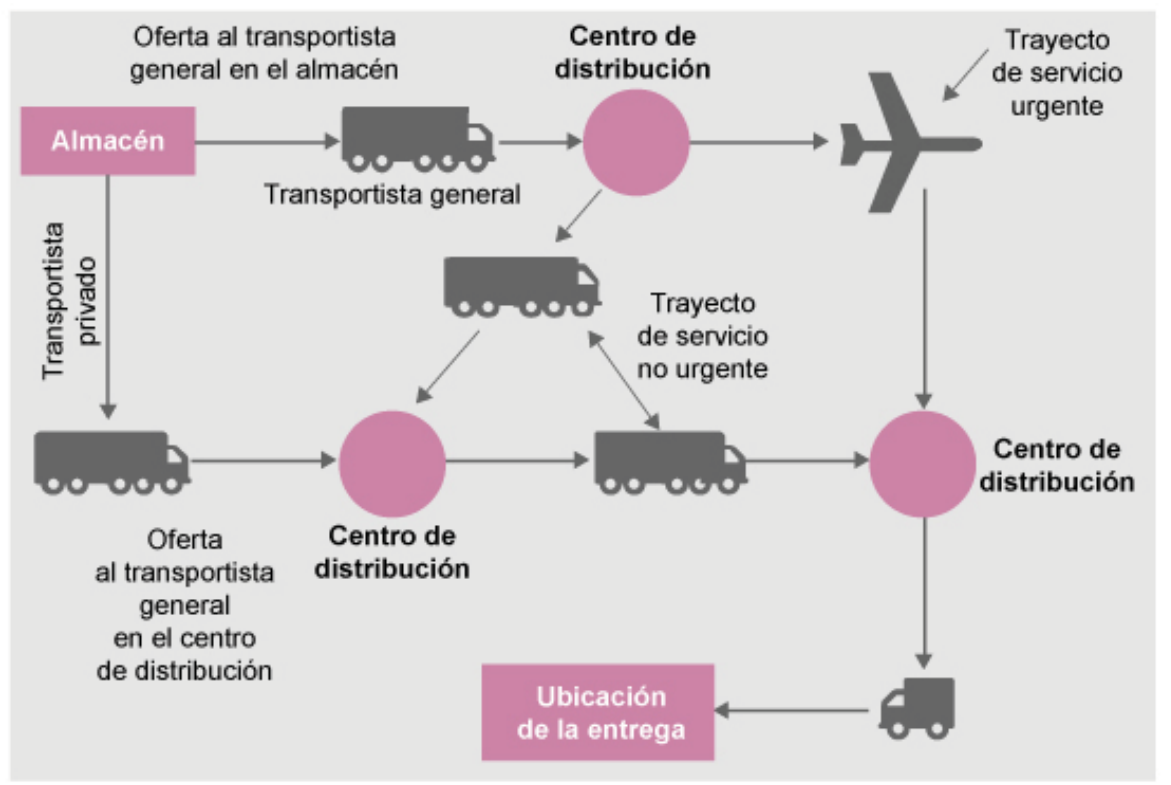

Fuente: elaboración propia a partir de Predictive Analytics Times

3. Nuevos sistemas de entrega al cliente. Sistemas de entrega automatizados

La posibilidad de automatizar los procesos logísticos permite incrementar la productividad y reducir los costes de mano de obra de una forma sensible, además de incrementar la seguridad, mejorar la calidad y acortar plazos. A lo largo de la cadena de suministro, se pueden automatizar desde procesos de picking o de entrega al cliente hasta el análisis y gestión de datos.

Así como la robotización de las tareas de almacén hace años que empezó, la automatización de la entrega al cliente es una tecnología bastante novedosa. McKinsey publicó un estudio hace unos meses en el que pronosticaba que, en el año 2025, el 80 \% de las entregas de eCommerce se harían mediante vehículos autónomos, incluyendo drones (por zonas rurales).

En estos momentos, los vehículos autónomos están en pleno desarrollo y todavía no es posible su uso para el transporte de mercancías; en cambio, la entrega a través de drones ya ha hecho el primer gran paso: el 14 de diciembre de 2016, se hizo la primera entrega comercial a través de un dron. Hizo falta 13 minutos desde el clic del comprador a la entrega al cliente, en Cambridge (Reino Unido). 
Otros estudios recientes corroboran que el $79 \%$ de los clientes estadounidenses muy probablemente pedirían entrega mediante drones, de los cuales un $73 \%$ estaría dispuesto a pagar un extra de 10 dólares por las ventajas que este tipo de entrega les ofrece.

Figura 7. Comparación del precio de entrega mediante dron frente a sistemas de distribución actuales

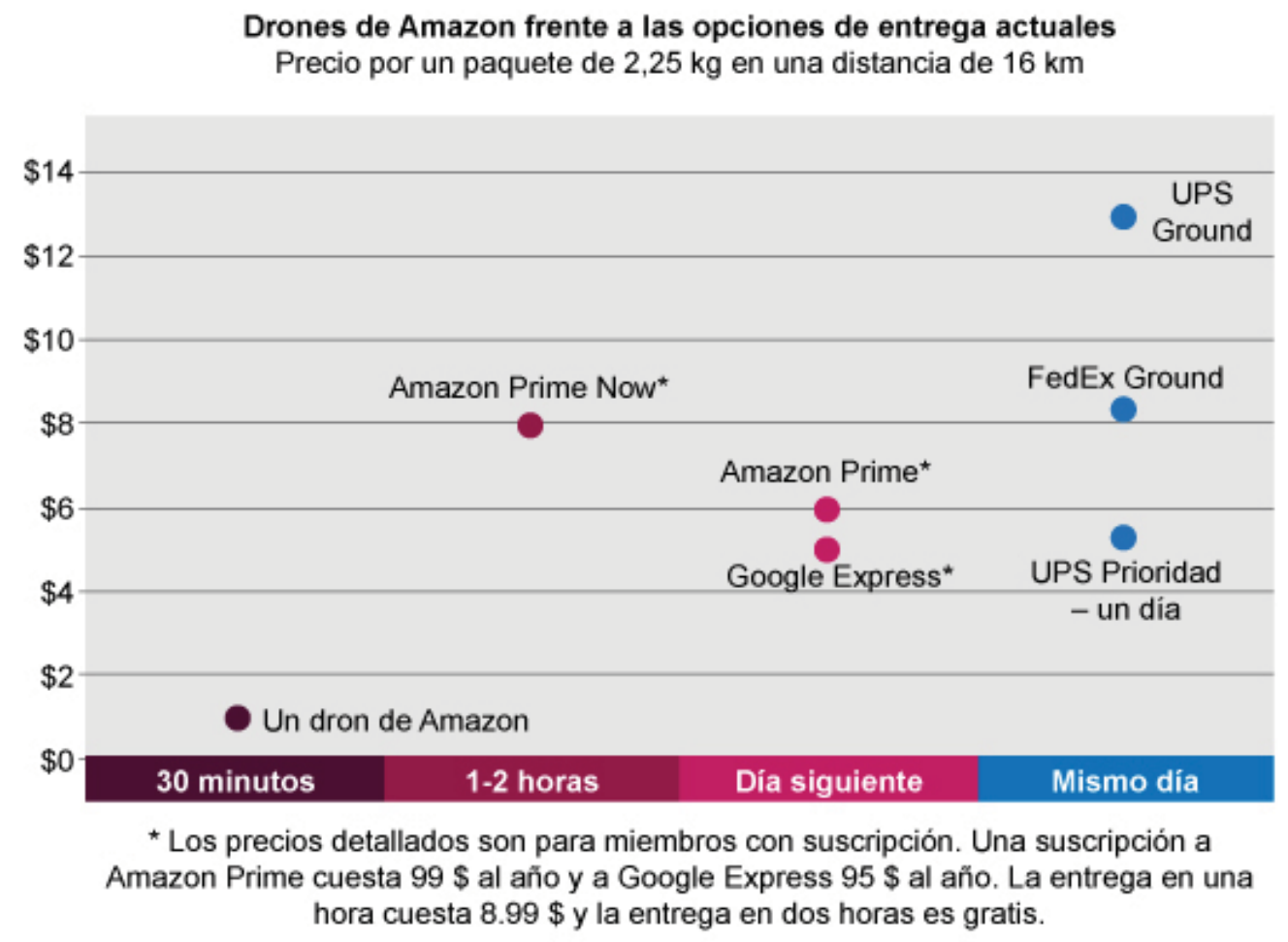

Fuente: https://www.flexport.com/blog/drone-delivery-economics/

Además de la aceptación creciente, otro aspecto que hay que tener en cuenta es la regulación del uso de esta tecnología, que tiene mucha diversidad de criterios: por ejemplo, mientras que actualmente la Administración Federal de Aviación de los Estados Unidos restringe el vuelo de artefactos no tripulados y no permite el vuelo si no hay visión directa, en el Reino Unido hay normas más flexibles que permiten realizar pruebas de larga distancia.

\subsection{D printing}

La visión más ambiciosa posiciona a la impresión 3D en un sustitutivo de las técnicas de manufactura tradicionales. No obstante, los costes de producción unitarios son difíciles de confrontar con las inversiones requeridas y/o el tiempo de producción ofrecidos por la impresión 3D. Más a corto plazo, la impresión 3D empieza a 
irrumpir en industrias especializadas en producción de elementos complejos y/o personalizados. En estos segmentos se empiezan a experimentar cambios en políticas de inventario y transporte. Concretamente, se experimenta un giro hacia la producción bajo demanda cerca del punto de entrega, reduciendo consecuentemente los niveles de inventario en centros de distribución intermedios.

Figura 8. Comparación de costes entre industria tradicional e impresión 3D

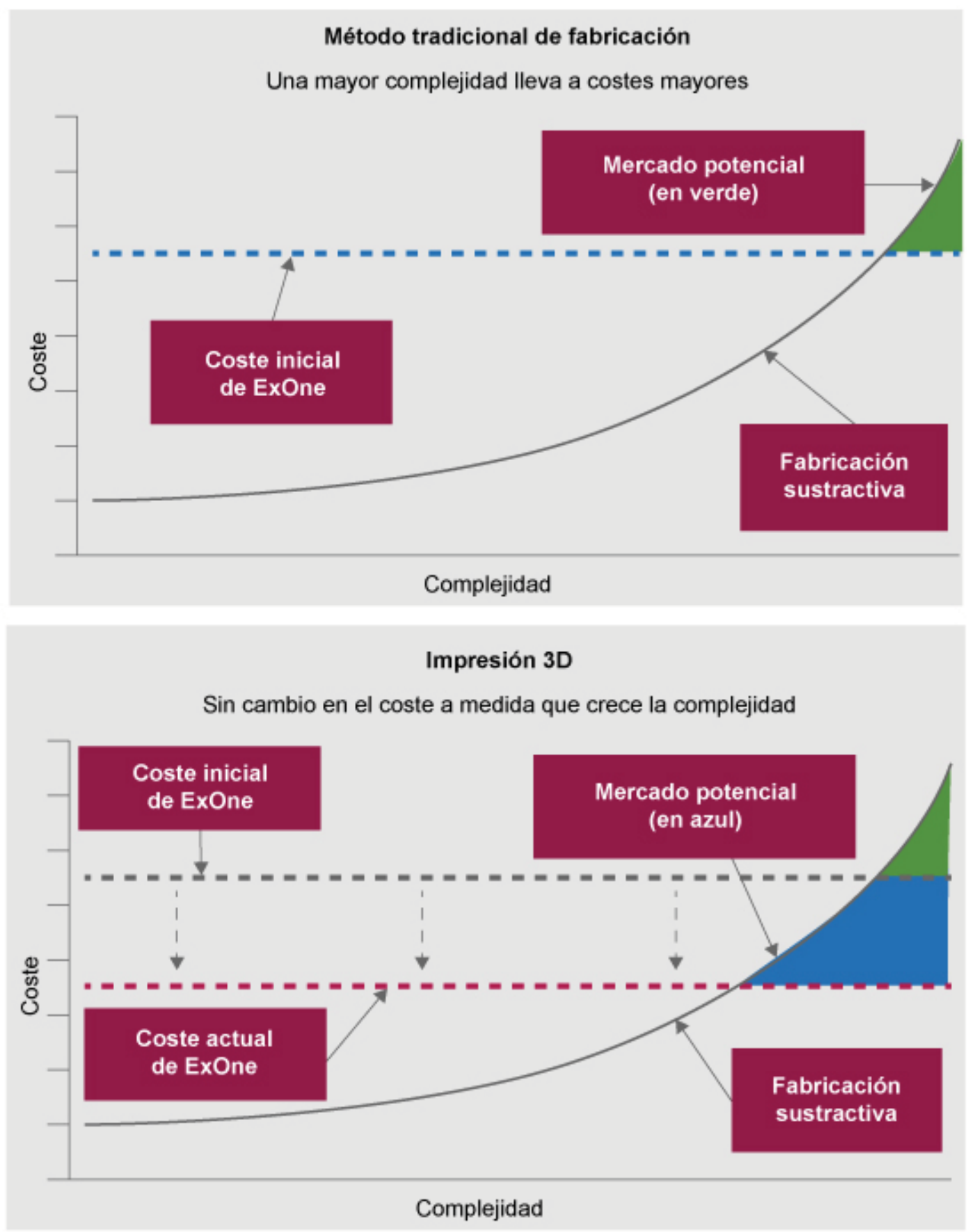

Fuente: Kholoudabdolqader https://commons. wikimedia.org/wiki/File\%3A3dvstra.png 
Este nuevo paradigma de producción bajo demanda puede cambiar las dinámicas entre productores y vendedores posicionando como actores clave a los proveedores logísticos. Los proveedores logísticos cuentan con una proximidad al cliente final que permitiría capturar las necesidades para la producción 3D y la personalización de los bienes a imprimir, pasando de almacenar y mover inventario a imprimir inventario en 3D según las necesidades del cliente final. Un híbrido entre centro tecnológico, punto de manufactura y de venta que exime al cliente y al distribuidor de los riesgos que comporta gestionar inventario.

A pesar de que la producción clásica está altamente descentralizada en ubicaciones caracterizadas por contar con costes reducidos, la gestión de la logística de la cadena de suministro global puede presentar hándicaps en este sentido debido a las tendencias alcistas en cuanto a costes asociados al transporte en los últimos tiempos. Entre otros muchos, en esta vertiente también el $3 D$ printing ofrece un gran potencial.

En todo caso, la impresión 3D ya es una realidad: además del uso muy extendido en los prototipos, empieza a extenderse ampliamente en el mercado de recambios. Este es el caso de Daimler AG (fabricante de los vehículos Mercedes, Smart, Mayback), que ofrece piezas de repuesto en todo el mundo a través de esta tecnología con plazos de entrega mucho más cortos.

\section{Conclusiones}

Existe en la actualidad una serie de tecnologías con una creciente relevancia dentro de la cadena de suministro: sistemas autónomos, 3D printing, internet of things (loT), inteligencia artificial y blockchain.

Su adopción y evolución requerirá de inversiones por parte de los diferentes actores de la cadena de suministro. Inversiones centradas no solo en el desarrollo o la adquisición de la tecnología, sino también en la redefinición de los procesos con los que las nuevas tecnologías interactuarán, transformando así la gestión de negocio. Se inicia una nueva etapa de transformación tecnológica con el objetivo de añadir valor a la experiencia del usuario en el proceso de compra mediante el logro de la excelencia operacional, personalizando servicios y productos, así como sus tiempos de entrega y la medida de lotes de compra.

Cualquier adopción tecnológica requerirá de un grado superior de especialización por parte de un sector que se caracteriza por una cierta rigidez, fruto de su elevado volumen de negocio, y por un fuerte enfoque operacional.

Asimismo, será necesaria la creación y la introducción de nuevos estándares para poder habilitar la adopción de tecnologías y su futuro crecimiento de una manera normada. 


\section{Datos de interés}

- En 2025 el 80 \% de los paquetes B2C serán entregados por vehículos autónomos, incluyendo drones, en zonas rurales (McKinsey).

- IDC Manufacturing Insights prevé, a finales del año 2020, que el $50 \%$ de todas las cadenas de suministro tendrán la capacidad de habilitar envíos directos a clientes finales y/o entrega a domicilio.

- La tasa de entrega fallida se sitúa en España entre el 20 \% y el 30 \%, según el Libro Blanco de la Logística de Adigital.

- Según una encuesta realizada en 2017 a doscientos directivos de la cadena de suministro de empresas de distribución norteamericanas, el 83 \% consideraba que la experiencia de cliente tenía que ser uno de los ejes principales de su estrategia, pero, en cambio, un $66 \%$ consideraba que no tenía las soluciones tecnológicas adecuadas para afrontar este reto, y un 79 \% decía que la eficiencia era todavía el principal objetivo o uno de los principales (ETFConvey).

\section{Referencias bibliográficas}

AGRAWAL, A.; GANS, J.; GOLDFARB, A.; (2017). «How Al will change strategy: a thought experiment». Harvard Business Review. <hbr.org>. H03XDI.

AHMED, S.; LIU, H.; WANG, S.; YANG, S. (2015). Showcasing Work by Amazon. com on Recommender System. CS 548 Spring 2015 Web Mining Showcase. Worcester Polytechnic Institute.

BUDET, J.; PÉREZ, A. (2018). «La logística como fuente de valor añadido al eCommerce». Oikonomics. N. ${ }^{\circ}$

CECERE, L. (2017). «Digital Transformation: It Takes a Village». Supply Chain Shaman.

CECERE, L. (2017). «If Only There Were More Choices». Supply Chain Shaman.

JOERSS, M.; SCHRÖDER, J.; NEUHAUS, F.; KLINK, C.; MANN, F. (2016). Parcel delivery: the future of last mile. McKinsey \& Company.

KIM, H. M.; GHIASI, B.; SPEAR, M.; LASKOWSKI, M.; LI, J.; (2017). «Online serendipity; The case for curated recommender Systems». Business Horizons. Vol. 60, n. ${ }^{\circ}$, págs. 613-620.

ORIOL, A. (2017). «Will you be a Supply Chain Disruptor or the Disrupted?». CSCMP Quarterly.

SAAR-TSECHANSKY, M. (2014). "Clustering Analysis; Data mining for Business intelligence». Teaching notes. McCombs School of Business. The University of Texas. Austin. 
WALKER, R.; JIWANI, R. (2016). «Reinventando el comercio electrónico: La apuesta de Amazon por el reparto de mercancías con vehículos no tripulados». Northwestern Kellogg School of Management. KEL931.

YEN, B. (2016). "The internet of things (IOT): Shaping the future of e-commerce». ACRC The University of Hong Kong Asia Case Research Centre. HK1063.

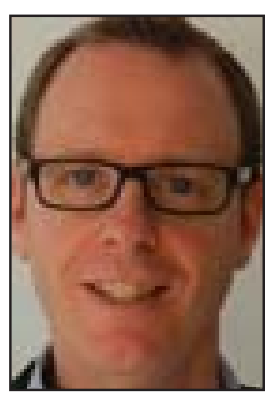

\section{Xavier Budet Jofra} xbudet@uoc.edu Profesor colaborador del máster de Dirección de operaciones y logística integral (UOC)

Ingeniero industrial superior por la Escuela Técnica Superior de Ingenieros Industriales de Barcelona (UPC). MBA por ESADE (Barcelona) y CSCP por Apics (Estados Unidos). Actualmente es director de Logística- Canal Online en La Sirena Alimentación Congelada S.L.U. También es profesor colaborador, tutor de proyectos en estudios de grado y máster relacionados con la cadena de suministro y autor de publicaciones de la UOC.

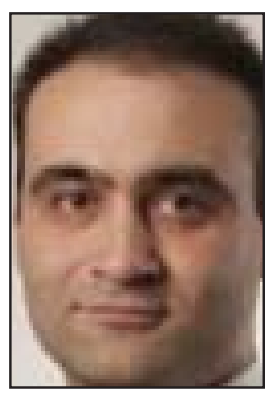

\section{Alexis Pérez Gómez} aperezgomez3@uoc.edu Profesor colaborador del máster de Dirección de operaciones y logística integral (UOC)

MBA por ESADE (Barcelona y McCombs Austin, Texas). Ingeniero industrial superior por la Escuela Politécnica Superior de la Universidad de Gerona. Ingeniero técnico en Informática de Sistemas por la Escuela Politécnica Superior de la Universidad de Gerona. Actualmente, es responsable de producción, abastecimiento de material y calidad de la actividad de reparaciones y apoyo al cliente de Europa, Medio Oriente y África en HP Inc. También es profesor colaborador, tutor de proyectos en estudios de grado y máster relacionados con cadena de suministro y autor de publicaciones de la UOC. 
Los textos publicados en esta revista están sujetas -salvo que se indique el contrario- a una licencia de Reconocimiento 3.0 España de Creative Commons. Podéis copiarlos, distribuirlos, comunicarlos públicamente y hacer obras derivadas siempre que reconozcáis los créditos de las obras (autoría, nombre de la revista, institución editora) de la manera especificada por los autores o por la revista. La licencia completa se puede consultar en http://creativecommons.org/licenses/by/3.0/es/deed.ca.

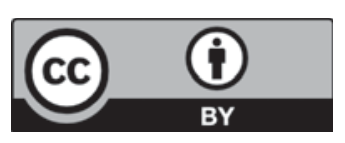

